\title{
Lipedema with genital lymphatic vesicles
}

\section{Asmae Abdelmouttalib, Mehdi Khallayoune, Nadia Ismaili, Karima Senouci}

\author{
Dermatology and Venereology Department, Mohamed V University in Rabat, Morocco
}

Corresponding author: Asmae Abdelmouttalib, MD, E-mail: abdelmouttalibasmae@gmail.com

Sir,

Lipedema (or the "pole leg" disease) is a rare and painful disorder of the adipose tissue. It mainly affects females and is generally misdiagnosed as lymphedema or obesity. It may progress to involve the venous system (venolipedema) or the lymphatic system (lympholipedema), adding to the difficulty in its diagnosis. Herein, we report a case of lipedema with genital lymphatic vesicles.

A 56-year-old female presented herself with a symmetrical increase in the volume of both lower limbs persistent since a young age, painful and with repetitive bruising. The patient reported the same condition in her 45-yearold sister. A clinical examination revealed a bilateral and symmetrical enlargement of the legs without the involvement of the feet, with a sharp demarcation between normal and anormal tissue at the ankle. The skin was firm in consistency, irregular, painful on pressure, and of a normal color. Stemmer's sign was absent (Fig. 1). A genital examination revealed papillomatous formations, which failed to whiten after the application of acetic acid and which corresponded to lymphatic vesicles and not to condylomas (Fig. 2). The patient was advised to lose weight, engage in regular physical activity with lymphatic drainage sessions, and wear compression stockings. Moreover, the lymphatic vesicles were not touched because of the risk of lymphorrhea.

Lipedema is considered to be a rare disorder of the adipose tissue. Its etiology is unknown and its pathophysiology is not clearly elucidated [1]. Lipedema exhibits sex-linked, X-linked, dominant or autosomal dominant inheritance [2]. The diagnosis of lipedema is quite frequently missed because of the clinician's usual unfamiliarity with this condition, because of the overdiagnosis of lymphedema, and because of its occasional misdiagnosis as obesity [3,4]. Lymphedema is commonly asymmetric, without associated pain

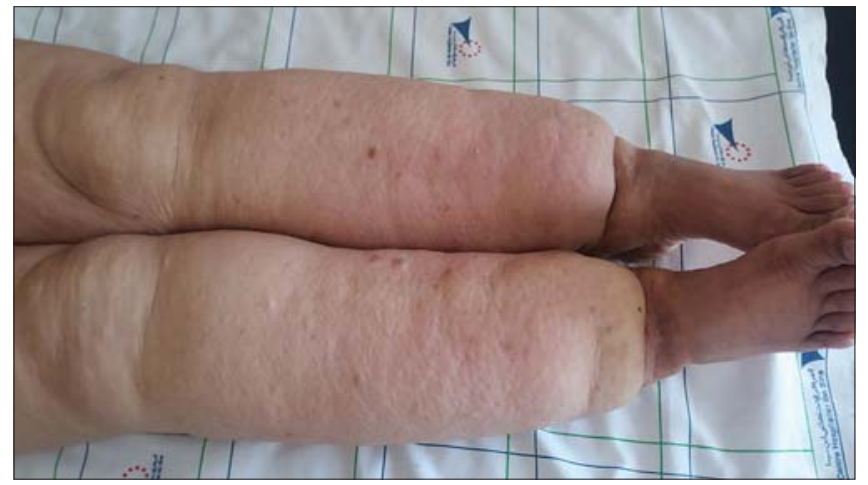

Figure 1: The "pole leg" presentation without the involvement of the feet.

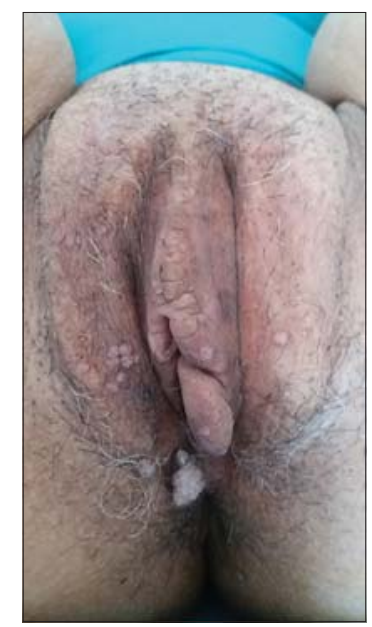

Figure 2: The lymphatic vesicles at the genital level that did not bleach after the application of acetic acid.

or bruising. Lipedema is bilaterally symmetrically distributed and is associated with pain, edema, and easy bruising. It commonly affects females after puberty but also during pregnancy and after menopause $[2,5]$. The disease progresses in three stages and the typical presentation is that of a female with bilateral "stovepipe" enlargement of the legs and without the involvement of the feet, with a sharp demarcation between normal and abnormal tissue at the ankle, referred to as the "cuff"

\footnotetext{
How to cite this article: Abdelmouttalib A, Khallayoune M, Ismaili N, Senouci K. Lipedema with genital lymphatic vesicles. Our Dermatol Online. 2021;12(2):222-223.

Submission: 06.12.2020; Acceptance: 21.02.2021

DOI: $10.7241 /$ ourd.20212.38
} 
sign. Lipedema is, however, a chronic progressive disease and advanced cases may deteriorate to involve either the lymphatic system (lympholipedema) or the venous system (venolipedema), or both, which adds to the confusion in the diagnosis. The diagnosis of lipedema is clinical and it is essential to recognize this unique entity in order to establish proper treatment [1]. Treatment involves maintaining a healthy weight, engaging in physical activity, wearing class 2 venous compression stockings, and, possibly, undergoing liposuction for esthetical reasons.

Lipedema is a pathological entity that poses a diagnostic problem, especially being misdiagnosed as lymphedema and, sometimes, obesity. Characteristics observed during the interrogation and clinical examination allow the diagnosis to be reached even if an association with the abnormalities of lymphatic drainage appears in some advanced cases, as in ours.

\section{Consent}

The examination of the patient was conducted according to the principles of the Declaration of Helsinki.
The authors certify that they have obtained all appropriate patient consent forms, in which the patients gave their consent for images and other clinical information to be included in the journal. The patients understand that their names and initials will not be published and due effort will be made to conceal their identity, but that anonymity cannot be guaranteed.

\section{REFERENCES}

1. Shavit E, Wollina U, Alavi A. Lipoedema is not lymphoedema: A review of current literature. Int Wound J. 2018;15:921-8.

2. Child AH, Gordon KD, Sharpe P, Brice G, Ostergaard P, Jeffery S, et al. Lipedema: An inherited condition. Am J Med Genet A. 2010;152A:970-6.

3. Fonder MA, Loveless JW, Lazarus GS. Lipedema, a frequently unrecognized problem. J Am Acad Dermatol. 2007;57(2 suppl):S1-3.

4. Godoy Mde F, Buzato E, Brigidio PA, Pereira de Godoy JM. Is lymphostasis an aggravant of lipedema? Case Rep Dermatol. 2012;4:222-6.

5. Okhovat JP, Alavi A. Lipedema: A review of the literature. Int J Low Extrem Wounds. 2015;14:262-7.

Copyright by Asmae Abdelmouttalib, et al. This is an open access article distributed under the terms of the Creative Commons Attribution License, which permits unrestricted use, distribution, and reproduction in any medium, provided the original author and source are credited.

Source of Support: Nil, Conflict of Interest: None declared. 